\title{
Intraductal Papillary Mucinous Neoplasia - a challenging diagnosis in a patient with paraneoplastic rheumatoid arthritis. Case report.
}

\author{
Ioana Grigorescu' ${ }^{1}$, Andrei Cismaru1, Romeo Chira ${ }^{2}$, Adrian Catinean2, Dan Lucian \\ Dumitrascu $^{1}$
}

${ }^{1} 2^{\text {nd }}$ Internal Medicine Department, ${ }^{2} 1^{\text {st }}$ Internal Medicine Department, "Iuliu Hatieganu” University of Medicine and Pharmacy, Cluj-Napoca, Romania

\begin{abstract}
Intraductal papillary mucinous neoplasias (IPMNs) of the pancreas are potentially malignant tumors, and associated also with extrapancreatic carcinomas. We present the case of a 80 year old man with IPMN and late onset of rheumatoid arthritis. The particularity of this case is the discordance between the clinical picture and the imaging worrisome features, together with the surgery-requiring mixed-type of IPMN, despite the late possible paraneoplastic onset of rheumatoid arthritis.

Keywords: intraductal papillary mucinous neoplasia, endoscopic ultrasonography, rheumatoid arthritis
\end{abstract}

\section{Introduction}

Intraductal papillary mucinous neoplasias (IPMNs) are potentially malignant intraductal epithelial neoplasms which consist of cystic dilatation of the main pancreatic duct (MD)-type, side branches (BD)-type or mixed-type [1]. They represent precursors of the invasive ductal adenocarcinoma of the pancreas and even for extrapancreatic (especially colorectal and gastric) malignancies [2]. For this reason the follow-up and systemic surveillance of patients is very important, even if they do not undergo surgery. According to the Sendai Consensus Guidelines, every recognized MD-IPMN and mixed-type IPMN should be treated with resection of the pancreas.

In this case report we describe a patient with IPMN and late onset of rheumatoid arthritis (RA).

Received 13.10.2014 Accepted 15.12.2014

Med Ultrason

2015, Vol. 17, No 1, 129-132

Corresponding author: Ioana Grigorescu, MD

2nd Internal Medicine Department

"Iuliu Hatieganu" University of Medicine and

Pharmacy, Cluj-Napoca, Romania

2-4 Clinicilor str

400006 Cluj Napoca, Romania

Email: ioanaduca2000@yahoo.com

Phone: 0040264591942

\section{Case report}

A 80 year old man, with a personal history of coronary artery disease, primary arterial hypertension, old ischemic stroke with left hemiparesis, recurrent urinary infections, operated prostatic adenoma, recent onset of RA (6 months before admission, treated with $15 \mathrm{mg} /$ week Methotrexate and $7.5 \mathrm{mg} /$ day Prednisone), and of hepatic cysts, was admitted for a neoplastic screening. His complaints consisted of inflammatory pain of the small joints of the hands and shoulders, and more than $30 \mathrm{~min}$ of morning stiffness. Additional, he had noted weight loss $(6 \mathrm{~kg}$ in the last 6 months), loss of appetite, aggravated constipation, asthenia, and intermittent pain in the right upper quadrant.

General physical examination revealed relatively good general condition, no fever, pallor, slight ulnar deviation of the hands, metacarpophalangeal arthritis bilateral; abdominal physical examination revealed pain in the right upper quadrant at deep palpation.

Laboratory data revealed: slight anemia (hemoglobin of $11.04 \mathrm{mg} \%$ ), moderate inflammatory syndrome, no cholestasis, leucocyturia, urine culture with E.Coli, normal serological tumoral markers (carcinoembryonic antigen CEA $1.70 \mathrm{ng} / \mathrm{ml}$, carbo- 


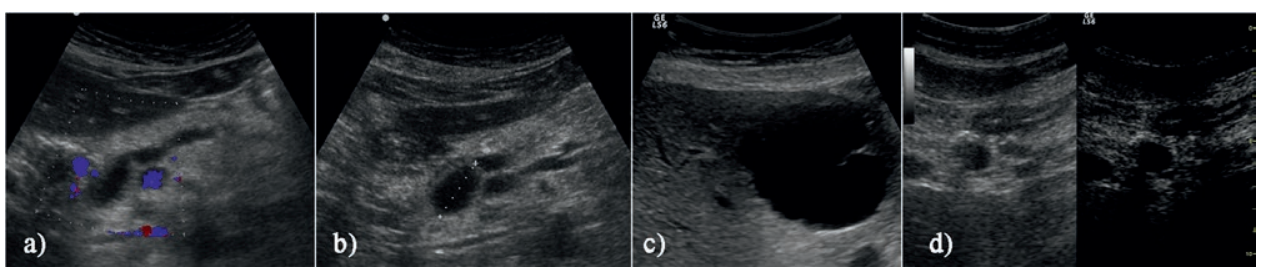

Fig 1. a) Colour-Doppler US: tortuous traiectory of the dilated Wirsung duct; b) Grey scale US: cystic dilatation in the pancreatic head with communication to the MPD; c) Grey scale US: hepatic cyst with septum; d) CEUS: nonenhancing pancreatic cysts

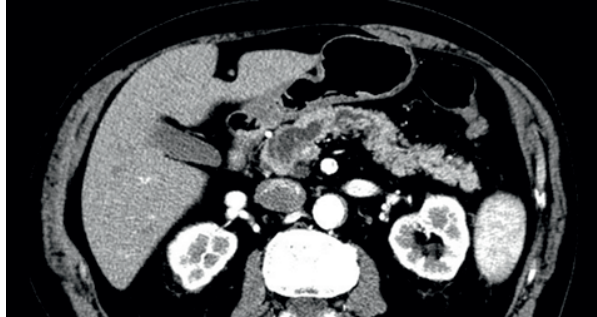

Fig 2. CT(arterial phase): dilated main pancreatic duct

hydrate antigen CA $19-9$ 15.09U/ml, prostate-specific antigen PSA $2.03 \mathrm{ng} / \mathrm{ml}$ ).

Colonoscopy revealed no tumoral masses, and upper gastrointestinal endoscopy had normal findings. ENT and urologic consultations excluded any tumor. Chest X-ray revealed no suspect mass. Grey scale ultrasound (US) described dilated Wirsung duct up to $7 \mathrm{~mm}$ (pancreatic body) and $1.2 \mathrm{~cm}$ (pancreas head) on its whole distance, with a tortuous traiectory (fig 1a) and a hypoechoic mass in the head of the pancreas of $2.3 / 1 \mathrm{~cm}$ in size, with hyperchoic walls, apparently communicating with the Wirsung duct (fig 1b). The US diagnostic suspicion was of ectasia of the Wirsung duct or neoplasia of the pancreas head. Associated, several well delimited cystic masses (the largest being of $5.7 \mathrm{~cm}$ in size) were seen in segments II, IV, V and VIII of the liver, the biggest one having a hyperechoic avascular septum (fig 1c), suggesting biliary cysts, with impossibility of complete exclusion of cystic metastases. One round hypoechoic lymph node (1.5 $\mathrm{cm}$ in size) in the liver hilum was detected. Contrast enhanced ultrasound (CEUS) (with $2.4 \mathrm{ml}$ Sonovue) was performed and a homogeneous enhancing of the head of the pancreas was noted with exception of the cystic masses in the head of the pancreas, which remained non-enhancing during the whole period of examination (5 minutes) (fig 1d); the liver cysts had also the same behaviour.

Abdominal CT scan revealed dilated Wirsung duct, with visible ectatic dilatations of the main pan-

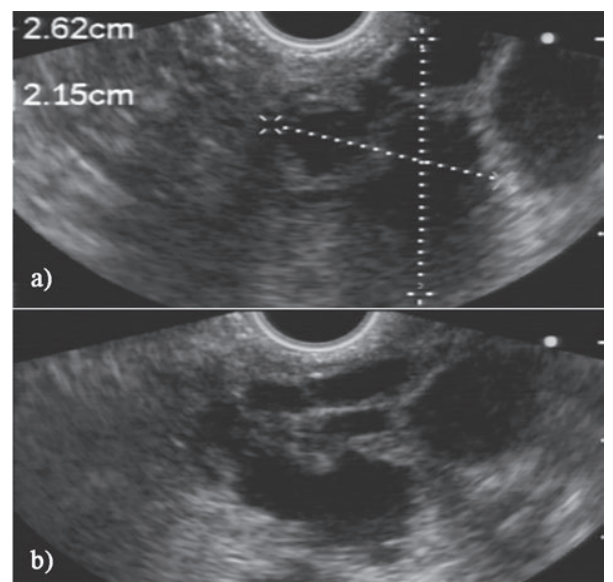

Fig 3. a) EUS: septated cysts of pancreatic head b) EUS: communication of the cyst with pancreatic ducts

creatic duct in the pancreas head (fig 2), but with no obvious tumoral mass.

We considered endoscopic ultrasound (EUS) as the next necessary step, which revealed several septated cysts (the largest $2.6 \times 2.2 \mathrm{~cm}$ in size) in the pancreatic head, having direct contact and communication with the Wirsung duct and its segmentary branches (fig 3), and thin wall and without any nodules of their walls, suggesting the diagnosis of IPMN; neither signs of pancreatic malignancy nor intrahepatic nodules had been found. High levels of tumoral markers (CA19-9 $70.6 \mathrm{U} / \mathrm{ml}$ and CEA $420 \mathrm{ng} / \mathrm{ml}$ ) in the specimen taken from the pancreatic cysts by means of EUS-fine needle aspiration (FNA) but without malignant cells at cytology were detected. Main biliary duct and papilla were described as normal in size and aspect.

The case was interpreted as a mixed-type IPMN of the pancreas, biliary cysts of the liver and paraneoplastic elderly-onset rheumatoid arthritis, establishing the necessity of surgery; at one month-follow-up, the patient was admitted with aggravated symptoms and progressive imaging features, but he still pleaded for conservative treatment. 


\section{Discussions}

Branch duct IPMNs were defined as an IPMN with a dilated branch and mixed IPMNs as a cystic lesion in the branch duct in combination with a mural nodule continuous with a dilated MD. Our case fits in the mixed-type IPMN after Choi's classification [3] with more cysts and diameter $>5 \mathrm{~mm}$ of the MD [4].

The diagnosis of IPMNs is more frequently made nowadays because of the improvement in imaging technologies; it includes radiographic (CT, magnetic resonance imaging-MRI, magnetic resonance cholangiopancreatography-MRCP) and endoscopic evaluation (endoscopic retrograde cholangiopancreatography-ERCP, EUS), positron emission tomography-PET, as well as serum tumor markers and molecular markers. These techniques with the possibility of performing fine-needle aspiration (FNA) and fluorodeoxyglucose (FDG)-PET can be used in order to diagnose and characterize IPMN [5].

Two-thirds of IPMN patients are men, with a peak age in the sixth decade.

Elderly-onset of rheumatoid arthritis, over 60 years, differs from the classic RA by a more equal gender distribution, a higher frequency of acute onset with systemic features, more frequent involvement of the shoulder, and higher disease activity, as in our case [6]. The patient's resistance to the treatment could be explained by the possible paraneoplastic etiology of the RA.

Regarding the clinical picture in IPMN, most patients have a long-standing history of recurrent acute pancreatitis or chronic obstructive pancreatitis (due to intermittent obstruction of the pancreatic duct by a mucus plug). None of these were present at our patient. Other possible symptoms include: diabetes, jaundice, back pain, and body weight loss. Many patients are however asymptomatic. One meta-analysis [4] specified that jaundice, abdominal pain, back pain, fever, weight loss, pancreatitis, or recent-onset diabetes are the most frequent symptoms attributed to cysts. Ferrone et al [7] classified cysts as asymptomatic if they were detected by imaging methods in patients with nonspecific abdominal pain (of nonpancreatic origin).

Eto et al [8] studied the biological significance of clinical malignant predictive markers, such as diameter of main pancreatic duct, cystic size and mural nodules. This study supports the positive correlation between an increased diameter of main pancreatic duct and high cell proliferative activity in IPMNs; dilatation of main pancreatic duct might predict the biologically high tumor proliferative activity. Serum levels of CA 19-9,
CEA and mucin-5AC (MUC5AC) may aid in differentiating between invasive and benign IPMN [9]. Serological tumoral markers were in normal range, but high levels of tumoral markers taken from the pancreatic cysts guided by FNA-EUS were detected in our patient.

Many patients with IPMN are found with concomitant cysts of the liver and kidney, some authors revealing the possibility of an underlying systemic cystic disorder [10], but with no statistical association.

The meta-analysis of Anand et al presented the risks of malignancy associated with individual cyst features including any of the features mentioned in the consensus guidelines for surgical resection: cyst size $>3 \mathrm{~cm}$, presence of mural nodules, dilatation of the main pancreatic duct $(>6 \mathrm{~mm})$ and main vs branch duct IPMN. Among all of these, the cyst size greater than $3 \mathrm{~cm}$ was associated most strongly with malignant IPMN. EUS with fine-needle aspiration (FNA) has an indication in patients with alarming features by some authors [11]. Our patient had no clinical and serological-biological predictive markers for malignancy, except weight loss, but problematic features at imaging methods (dilatation of the main pancreatic duct) and moderate elevated tumoral markers at EUS-FNA.

The international guidelines for IPMNs management state that the indications for surgery include the presence of mural nodules, but do not specify their diagnostic criteria. As EUS-FNA cytology has a low histologic correlation, Ohno et al consider that CE-EUS is the most reliable and appropriate method to base the indications for surgery on its findings [12]. As cytology was negative in our case, but there were positive tumoral markers at EUS-FNA, CE-EUS should have beenperformed, but the patient refused further investigation.

Observation is considered an option in patients with BD-IPMN with MNs of less than $10 \mathrm{~mm}$ in height [13]. This conservative attitude (for years) is also possible if MPD-IPMN patients are not suitable for surgery, MPD diameter $<15 \mathrm{~mm}$, negative cytology, and no mural nodules.

The particularity of this case is the discordance between the clinical picture and the imaging worrisome features, together with the surgery-requiring mixedtype of IPMN, despite the late onset of rheumatoid arthritis.

In conclusion IPMN is not a rare condition of the pancreas and can be suspected already at grey scale US and confirmed by EUS. Therefore cystic masses of the pancreas require special biological and imaging algorithms and follow-up strategies, especially if the clinical picture is atypical and might suggest a malignancy. 


\section{References}

1. Terris B, Ponsot P, Paye F, et al. Intraductal papillary mucinous tumors of the pancreas confined to secondary ducts show less aggressive pathologic features as compared with those involving the main pancreatic duct. Am J Surg Pathol 2000; 24: 1372-1377.

2. Choi MG, Kim SW, Han SS, Jang JY, Park YH. High incidence of extrapancreatic neoplasms in patients with intraductal papillary mucinous neoplasms. Arch Surg 2006; 141: 51-56.

3. Choi BS, Kim TK, Kim AY, et al. Differential diagnosis of benign and malignant intraductal papillary mucinous tumors of the pancreas: MR cholangiopancreatography and MR angiography. Korean J Radiol 2003; 4: 157162.

4. Anand N, Sampath K, Wu BU. Cyst features and risk of malignancy in intraductal papillary mucinous neoplasms of the pancreas: a meta-analysis. Clin Gastroenterol Hepatol 2013; 11: 913-921.

5. Yamada Y, Mori H, Matsumoto S. Intraductal papillary mucinous neoplasms of the pancreas: Correlation of helical CT and dynamic MR imaging features with pathologic findings. Abdom Imaging 2008; 33: 474-481.

6. van Schaardenburg D. Rheumatoid arthritis in the elderly. Drugs Aging 1995; 7: 30-37.
7. Ferrone CR, Correa-Gallego C, Warshaw AL, et al. Current trends in pancreatic cystic neoplasms. Arch Surg 2009; 144: 448-454.

8. Eto T, Hayashi H, Kuroki H, et al. Association of dilated main pancreatic duct with biological high proliferative activity in intraductal papillary mucious neoplasm. J Clin Oncol 2012; 30: Abstract 187.

9. Fritz S, Hackert T, Hinz U, Hartwig W, Büchler MW, Werner J. Role of serum carbohydrate antigen 19-9 and carcinoembryonic antigen in distinguishing between benign and invasive intraductal papillary mucinous neoplasm of the pancreas. Br J Surg 2011; 98: 104-110.

10. Sato Y, Mukai M, Sasaki M, et al. Intraductal papillary-mucinous neoplasm of the pancreas associated with polycystic liver and kidney disease. Pathol Int 2009; 59: 201-204.

11. Varadarajulu S, Fockens P, Hawes RH. Best practices in endoscopic ultrasound-guided fine-needle aspiration. Clin Gastroenterol Hepatol 2012; 10: 697-703.

12. Ohno E, Hirooka Y, Itoh A, et al. Intraductal papillary mucinous neoplasms of the pancreas differentiation of malignant and benign tumors by endoscopic ultrasound findings of mural nodules. Ann Surg 2009; 249: 628-634.

13. Kobayashi G, Fujita N, Maguchi H, et al. Natural history of branch duct intraductal papillary mucinous neoplasm with mural nodules: a Japan Pancreas Society multicenter study. Pancreas 2014; 43: 532-538. 